\title{
A YOLO and convolutional neural network for the detection and classification of leukocytes in leukemia
}

\author{
Shakir Mahmood Abas ${ }^{1}$, Adnan Mohsin Abdulazeez ${ }^{2}$, Diyar Qader Zeebaree ${ }^{2}$ \\ ${ }^{1}$ Computer Sciences Department, Cihan University-Duhok, Duhok, Iraq \\ ${ }^{2}$ Research Center, Duhok Polytechnic University, Duhok, Iraq
}

\begin{tabular}{l} 
Article Info \\
\hline Article history: \\
Received Apr 9, 2021 \\
Revised Oct 25, 2021 \\
Accepted Nov 21, 2021 \\
\hline
\end{tabular}

Keywords:

CAD3

CNN

Leukemia

WBCs classification dataset

WBCs detection dataset

YOLO

\begin{abstract}
The developing of deep learning systems that used for chronic diseases diagnosing is challenge. Furthermore, the localization and identification of objects like white blood cells (WBCs) in leukemia without preprocessing or traditional hand segmentation of cells is a challenging matter due to irregular and distorted of nucleus. This paper proposed a system for computer-aided detection depend completely on deep learning with three models computeraided detection (CAD3) to detect and classify three types of WBC which is fundamentals of leukemia diagnosing. The system used modified you only look once (YOLO v2) algorithm and convolutional neural network (CNN). The proposed system trained and evaluated on dataset created and prepared specially for the addressed problem without any traditional segmentation or preprocessing on microscopic images. The study proved that dividing of addressed problem into sub-problems will achieve better performance and accuracy. Furthermore, the results show that the CAD3 achieved an average precision (AP) up to $96 \%$ in the detection of leukocytes and accuracy $94.3 \%$ in leukocytes classification. Moreover, the CAD3 gives report contain a complete information of WBC. Finally, the CAD3 proved its efficiency on the other dataset such as acute lymphoblastic leukemia image database (ALL-IBD1) and blood cell count dataset (BCCD).
\end{abstract}

This is an open access article under the CC BY-SA license.

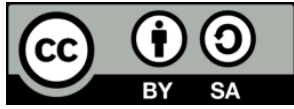

\section{Corresponding Author:}

Shakir Mahmood Abas

Computer Sciences Department, Cihan University-Duhok

Duhok, Iraq

Email: shakr.abbas@duhokcihan.edu.krd

\section{INTRODUCTION}

There are three main components of blood; red blood cells (RBC), white blood cells (WBC) and platelets. Microscopic test of blood smears plays an important role to diagnose many blood diseases. The white blood cells is the important component in human immune system [1]. There are four main types of white blood cells called lymphocytes, monocytes, neutrophils and eosinophil as shown in Figure 1 [2]. The number of white blood cells for each types and the total number of WBCs give us important information about the human health status. Moreover, it facilitates diagnosis of many blood diseases such as leukemia [3]. According to the type of infected cells and severity level, leukemia was divided into four main types: acute myeloid leukemia (AML), acute lymphoblastic leukemia (ALL), chronic myeloid leukemia (CML), and chronic lymphocytic leukemia (CLL) [2].

However, detection and classification of white blood cells from microscope images without preprocessing by deep learning is a challenge due to the lack of data, noise in resolution, irregular shape and color as they are from different sources. Traditionally, researchers used machine as an assistant for human to analysis medical images. The systems were consist of two main steps: features extraction and learning 
algorithms. In other side, many studies used image pre-processing as a main step for segmentation, feature engineering and manually object extraction to obtain high performance of detection and classification [4].

In other hand, some researchers mix between traditional techniques and deep learning to diagnosis some diseases and medical images analysis [5]-[7]. As first step the researchers prepare dataset to be ready for learning algorithms, this step includes preprocessing which means to apply some functions on data such as segmentation and filtration. The second step input data to deep learning algorithms such as convolutional neural network $(\mathrm{CNN})$ that is used for classification, recognition and detection.

Recently, the studies try to depend on deep learning algorithms to analyze medical images and diseases diagnosing [8]-[10]. The algorithms that used in this respect were such as region-based CNN (R-CNN), single shot detection (SSD) and you only look once (YOLO) for detection. Also fully convolutional network (FCN) and U-Net used for segmentation while the artificial neural network (ANN) and CNN used for classification [11], [12]. Moreover, the diagnosis by computer system that depends on deep learning is a great challenge. Deep learning assists to build computer aided design (CAD) system to do that automatically [13]. The main problems of this research are The use of preprocessing or segmentation before feeding the data into system [11], [14]. How to extract irregular white blood cells from the images that took from different sources. The difficulty of classification the irregular shape of white blood cells due to the vast changes in cells shape.

The paper amis using only deep learning approach without any traditional segmentation or preprocessing for detection and classification of white blood cells in leukemia. This paper is structured as: section 2 the background and related works. Section 3 the proposed systems architecture. Section 4 data acquisition and augmentation. Section 5 discussing the results and evaluating the CAD3 systems, and section 6 is conclusion and mentioning important points.

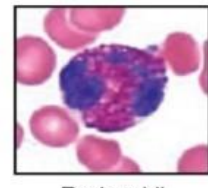

Eosinophil

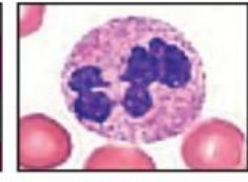

Neutrophil

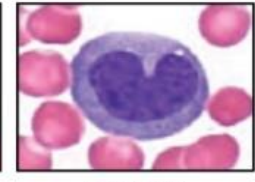

Monocyte

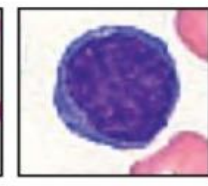

Lymphocyte

Figure 1. Types of white blood cell

\section{BACKGROUND AND RELATED WORK}

The rapid advancement of computer technology provides building of ANN with deep architectures for supervised and unsupervised learning such as self-driving cars and cancer detection [15]. $\mathrm{CNN}$ is considering one of the most common categories of deep learning that especially used for highdimensional data such as images and videos. The CNN is widely used for images classifications and objects detections [16], [17].

\subsection{Classification}

The terms CNN refer to a convolutional neural network which are case of deep learning neural networks that used mainly for classification [18]. The main different between ANN and CNN is convolution operation. The convolutional layer learn local small area with two dimensions while ANN layer learns global input space [19]. In Figure 2 the common architecture of CNN. The CNN consist of a set of layers and each layer consists of computational elements called neurons [20], [21]. However, there are three main layers used in $\mathrm{CNN}$, convolutional layers, pooling layers, and fully connected layers in addition, there are other optional layers can use in CNN such as batch normalization and dropout layer [14].

The convolutional layer is used to extract feature map by applying kernels over all input image. The kernel is small pieces of receptive field which consist of a set of learnable parameters [21], [22]. During forward pass process, each kernel convoluted with input image from top left into bottom right. The sliding process of kernel on input image is known as stride. Often, after the convolutional operation the feature map pass through batch normalization layer which normalize the gradients propagating, activations and make training of network easier [23], [24].

The pooling layer is the other important building block in $\mathrm{CNN}$ and it is filter with two dimensional. The filter slides on each feature map. The function of pooling layer is reducing the spatial size of the features maps [25]. There are several types of pooling layer such as max-pooling, min-pooling and average pooling. The max-pooling is common one used in CNN, usually with filter size $2 \times 2$ and stride of 2 . Thus, the output of max-pooling layer is containing the important features of previous feature map [7], [26]. Finally, fully 
connected which typically placed at end of network, this layer consists of neurons and activation function which provide the classification decision [27].

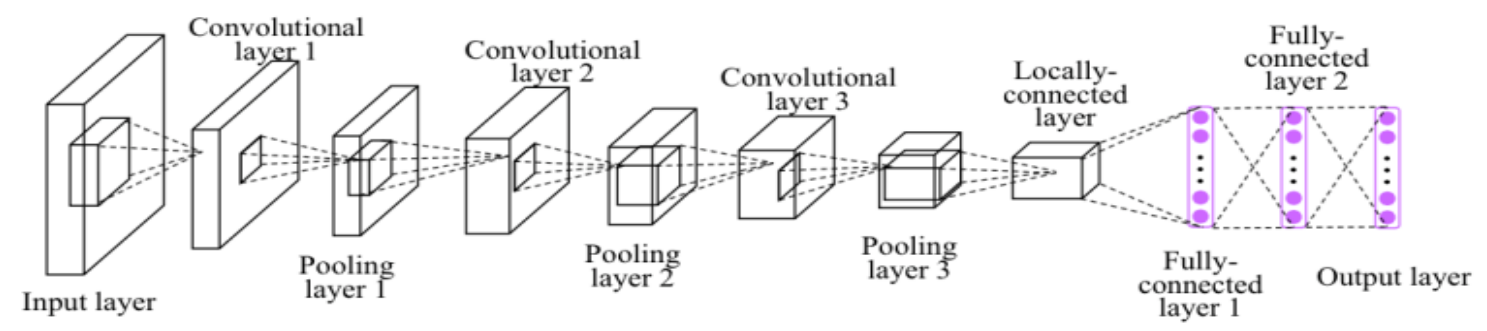

Figure 2. The main layers of $\mathrm{CNN}$

\subsection{Object detection}

The object detection is other approach depend on the deep learning that automatically locating interesting objects in a given image. In other word, object detection aims to identify the location of the objects in images [28]-[30]. In contrast, the object detection generates features map based on regional level of the image by drawn bounding box around the objects [31], [32].

There are many algorithms used for object detection such as R-CNN family and YOLO family. This paper focus on YOLO v2 algorithm [33], [34]. YOLO is popular detection algorithms used totally different approach than based-CNN. The first step of YOLO is dividing the input image into KxK grid cells and each cell is responsible for detect the object when the center of the objects falls into. YOLO provide the object localization and classification in each grid cell for one object. The limitation of YOLO v1 solved by YOLO v2 which focused basically on improving localization, average precision and accuracy [26], [35], [36]. Also, YOLO v2 use batch normalization which improves better accuracy and high resolution classifier. In Figure 3 the YOLO v2 architecture. In addition, YOLO v2 used anchor boxes which allows detecting more than one object in same grid cell [37]. The YOLO v2 used pre-trained network to extracts feature map such as (Alexnet, ResNet 50, and VGG16) [36]. The proposed system used the custom pre-trained network which we explain in the next section.

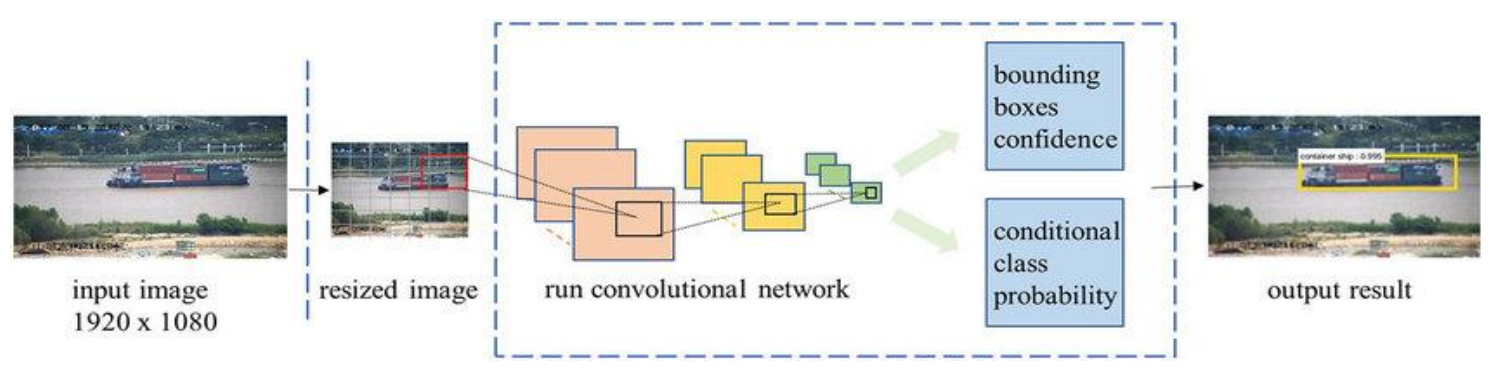

Figure 3. Example of YOLO v2 architecture

The extraction feature map pass through transform layer which responsible of organizing the feature map and creating YOLO v2 transform layer object to improves the network stability by determining location predictions [22], [38]. The YOLO v2 output layer is the last layer which responsible of determining and providing location of defined bound box for the target objects [39]. Moreover, this layers contain some properties such as loss function, anchor box and classes [40]. In addition, the YOLO v2 used mean suared error (MSE) loss function. This function is responsible of optimizing loss between predicted bounding boxes and the ground truth for refined bound box locations of output layer of YOLO v2 during training process.

Recently, many researchers used several methods and techniques in medical aspect, especially images analysis, Their aims were extract the main feature of images which was used in automatic diagnosing of diseases. Ghane et al. [38] the authors used segmentation technique as a main step by applying combination of modified watershed algorithms, thresholding and kmeans clustering. The proposed system consisted of three main stages; segmentation of WBCs, extraction of nuclei and separation of overlapping 
cells and nuclei. The image divides into four various regions which were red blood cells, nucleus of WBC, cytoplasm and background. Moreover, the author used preprocessing and morphological operations. In addition, applied some filters such cyan, magenta, yellow, and black (CMYK) color space, Otsu thresholding for pre-processing. Finally, for the nucleus segmentation used some algorithms such as k-mean algorithm. The system achieved similarity measures, precision, and sensitivity respectively were $92.93 \%, 97.41 \%$, and $93.78 \%$ for white blood cell segmentation. The proposed system was applied on 431 WBCs as dataset. Safuan et al. [41] mainly depended on the traditional segmentation to separate white blood cells by using system called computer aided system (CAS). This system consisted of four main stages, The first step was color space correction, in which the $\mathrm{L}^{*} \mathrm{a} * \mathrm{~b}$ color space is used to reduce the correlation between images channels. The key word of this step is making four templates of images instead of the original image. The second step is segmentation of white blood cells by using Otsu thresholding, CMYK and hue, saturation and value (HSV) on color space image. The third step is morphological filter which means eliminate the noises in the segmented image by applying two kinds of filters; connected component labeling (CCL) and morphological operation. Finally, the system counted and identified the white blood cells by using circle hough transform (CHT) method. This method counted the white blood cells by finding the circle for series range of radius from minimum to maximum to differentiate between white blood cells and other objects. WBC segmentation accuracy which was $96.92 \%$.

Aliyu et al. [42] proposed two systems to segment and classify the red blood cells basing on the shape of cells in images, the red blood cells were classified into normal and abnormal. The first system used support vector machine (SVM) to classify extracted feature in red blood cells images, the classifier based mainly on shape features. This method depended on weights absolute values of a linear classifier that trained on entire features set. The second system used deep neural network that learns on images directly by using cropped cells for classification tasks. In addition, images acquired from two database which contained of 105 and 250 images of red blood cells and the images were resized to $256 \times 256$. The system achieved accuracy $67 \%$ and $83 \%$. Pansombut et al. [43] proposed system based on machine learning that used convolutional neural network to classify white blood cells in an ALL in three classes and compared them with other traditional machine learning, multilayer perceptron (MLP), SVMs and random forest. The proposed CNN architecture consisted of seven layers, three layers were convolution and used 32 kernel with size 3 in first and second layer and 64 kernels with size 3 was used in third layer. In addition, two max pooling layers with size 2 applied to reduce the image size into half. The sixth layer was flattening layer in which multidimensional array converted into one-dimensional array. The seventh layer was fully connected layer followed by rectified linear unit (ReLU) activation function and dropout layer. The dataset were contained 363 color images with size $256 \times 256$ and the system achieved accuracy above $80 \%$.

Hung et al. [44] used deep learning for detecting red blood cells in malaria disease by using faster region CNN (faster R-CNN). The dataset that used was tacked from four patients in Brazil. Firstly, the images passed through region proposal network (RPN) which proposed the bounding box of object while the second stage classified bounding box to detect object. In this stage identified bound box around objects in image as red blood cells or other objects. The faster R-CNN based on AlexNet which consisted of 7 layers to identify red blood cells, white blood cells and platelets. After this stage the extracted red blood cells by Faster R-CNN fed into AlexNet again to classify red blood cells and the system achieved 59\% accuracy. Alam and Islam [45] proposed system based on deep learning for counting and identification three kinds of blood cells, the system used YOLO and GoogLeNet as pre-trained network. The YOLO framework trained on blood cell count dataset (BCCD) dataset which consisted of 364 blood images after modified configuration to detect and count blood cells automatically. The YOLO framework received image with size $448 \times 448$ then divided into $7 \times 7$ grid cell, two bounding boxes were used for each grid cell for prediction of blood cells when object center fell into grid cell. In addition, k-nearest neighbor (KNN) and intersection over union (IOU) used to remove multiple counting of the same object, the system achieved mean average precision map $62 \%$.

\section{THE PROPOSED COMPUTER-AIDED DETECTION SYSTEMS (CAD3)}

The localization and classification of white blood cells in leukemia require several stages starting from input step into the predicted output step in proposed system. The proposed CAD3 system consist of three main models and each model used individually to performing special purpose. The first model is used to detect the WBC of the input image then the second model is responsible to classify WBC and finally, classified WBC is visualize in original input image by third model as shown in Figure 4. 


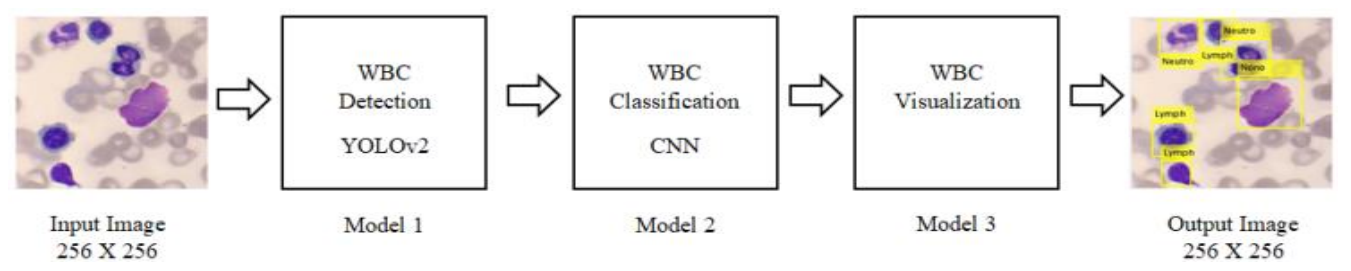

Figure 4. Global view of CAD3 system

\subsection{WBC detection model YOLO v2}

This model is used deep learning approaches to detect WBC instead of conventional segmentation and mainly consist of two parts; CNN and YOLO v2 layers. Moreover, this model is used to extract WBC of the images in dataset. And as it known, YOLO v2 relies on a pre-trained CNN to extract features map, a suitable CNN was created for this purpose. The CNN network is experimentally determined the suitable architecture of CNN as pre-trained network. Experimentally, the network with small and increasing number of features map improve better performance. The proposed CNN architecture is detailed in Table 1.

The feature extraction layer that used in YOLO v2 is most effective when width and height of feature map are between 8 and 16. The sixth convolutional layer in network was used as features map extraction and connected to YOLO v2 layers. However, the detection model YOLO v2 consist of 32 layers. The first layer is input layer which receive image with size $256 \times 256$. In addition, there are 9 convolutional layers, the first sixth layers used to extract features map with kernel size $(3 \times 3)$ and stride 1 , the YOLO v2 add convolutional layer with kernel $(3 \times 3)$ and stride 1 to organize the feature map and last convolutional layer is used kernel with size $(1 \times 1)$ which used to decrease the filter space channels. Furthermore, all convolutional layers are followed by Batchnornaliztion and ReLu nonlinear activation function, except of the final convolutional layer which is followed by YOLO v2 transform layer. In addition, four max pooling layers are used to reduce dimensions of feature map. Finally, the last layer in YOLO v2 detector network is YOLO v2 output layer which used MSE loss function and anchor box, the architecture of detection model is illustration in Figure 5. Detection model use 9 anchor with various shapes. The shapes of anchor box estimated from the training dataset.

Table 1. Proposed CNN as pre-trained for detection model

\begin{tabular}{clccc}
\hline Layer & Layer Type & Layer Output & Kernel Size & Stride \\
\hline 1 & Convolution & $256 \times 256 \times 16$ & $3 \times 3$ & 1 \\
2 & Max Pooling & $128 \times 128 \times 16$ & $2 \times 2$ & 2 \\
3 & Convolution & $128 \times 128 \times 32$ & $3 \times 3$ & 1 \\
4 & Max Pooling & $64 \times 64 \times 32$ & $2 \times 2$ & 2 \\
5 & Convolution & $64 \times 64 \times 64$ & $3 \times 3$ & 1 \\
6 & Convolution & $64 \times 64 \times 128$ & $3 \times 3$ & 1 \\
7 & Max Pooling & $32 \times 32 \times 128$ & $2 \times 2$ & 2 \\
8 & Convolution & $32 \times 32 \times 128$ & $3 \times 3$ & 1 \\
9 & Max Pooling & $16 \times 16 \times 128$ & $2 \times 2$ & 2 \\
10 & Convolution & $16 \times 16 \times 256$ & $3 \times 3$ & 1 \\
11 & Max Pooling & $8 \times 8 \times 256$ & $2 \times 2$ & 2 \\
12 & Fully Connected & 100 & - & - \\
13 & Fully Connected & 2 & - & - \\
\hline
\end{tabular}

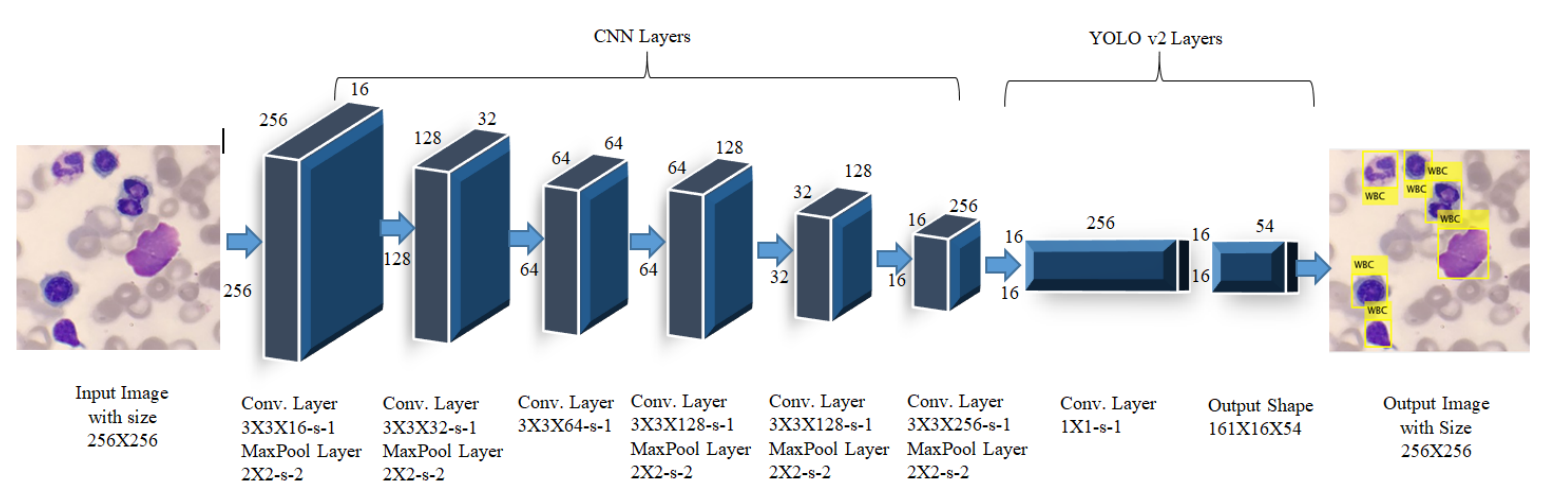

Figure 5. Detection model architecture 


\subsection{WBC classification model CNN}

This model is the second stage of CAD3 system which is also consider other main part of the system. Moreover, this model based on CNN which its functions is classification. After WBC is detected by the first model (YOLO v2 detector), the WBC images have different sizes pass into second model (classification model) to classify WBC into its labeled class. For this purpose, we build suitable CNN network that consists of multilayers and receives image with size $64 \times 64$. The chosen size of input images is average of WBC images size in dataset. The CNN consist of main three kinds of layers: convolutional layers, pooling layers, and fully connected layers as shown in Figure 6. In addition, other layers used such as patch normalization layer, ReLu activation function layers and dropout layer.

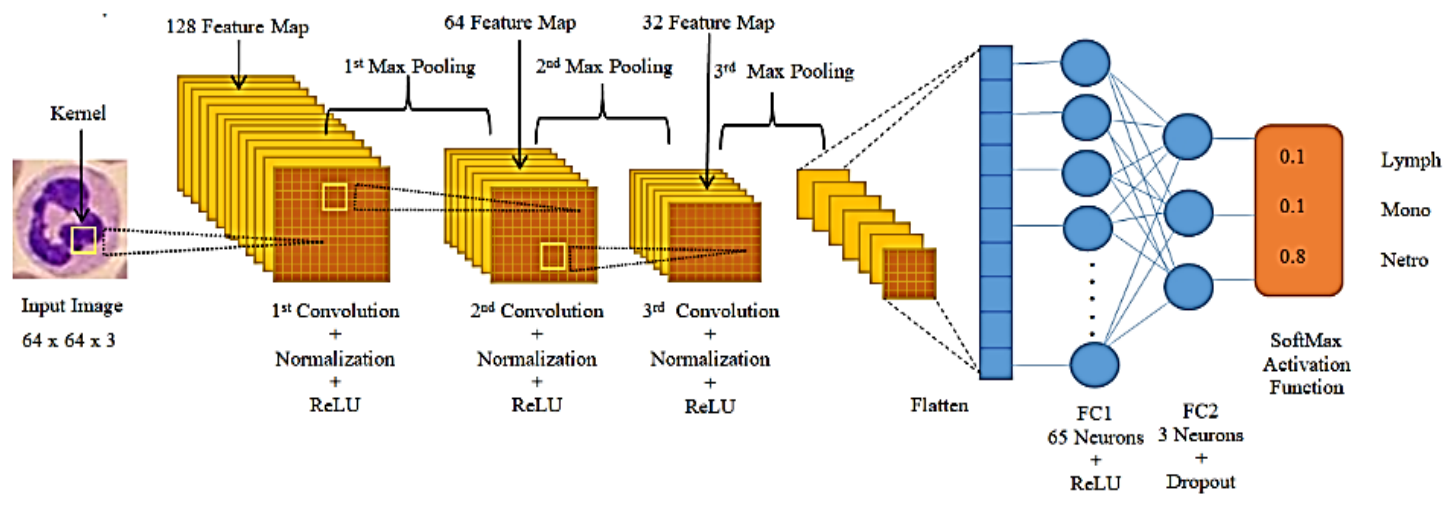

Figure 6. The architecture of CNN model

Three convolutional layers are used in $\mathrm{CNN}$ model. The first layer is receiving color WBC image with size $64 \times 64$ and then applying 128 filters (kernels) with size $3 \times 3$. Each filter slides on the image from lift top to right bottom by one pixel, this process called (stride). However, to keeping size of feature map output of convolutional layer equal to input image, zero-padding is used which means add one pixel around input image with value equal to zero before convolution process. The output of the first convolutional layer is 128 features map with size $64 \times 64$. The second convolutional layer is received feature map with size $32 \times 32$, and then 64 filters are applying which is the half of filters used in the first convolutional layer. Also, zero-padded is used with 1 stride. The output of this layer is 64 features map with size $32 \times 32$. The third convolutional layer is the last layer and it is the same previous convolutional layer but the different is size of received feature map. The size of input feature map of this layer is $16 \times 16$. Also, this layer is used 32 filters. The Table 2 shown main layers of proposed CNN in second model.

\begin{tabular}{clccc}
\multicolumn{6}{c}{ Table 2. Details of main important layer of CNN model } \\
\hline Layer & Layer Type & Layer Output & Kernel Size & Stride \\
\hline 1 & Convolution & $64 \times 64 \times 128$ & $3 \times 3 \times 3$ & 1 \\
2 & Max Pooling & $32 \times 32 \times 128$ & $2 \times 2$ & 2 \\
3 & Convolution & $32 \times 32 \times 64$ & $3 \times 3 \times 128$ & 1 \\
4 & Max Pooling & $16 \times 16 \times 64$ & $2 \times 2$ & 2 \\
5 & Convolution & $16 \times 16 \times 32$ & $3 \times 3 \times 64$ & 1 \\
6 & Max Pooling & $8 \times 8 \times 32$ & $2 \times 2$ & 2 \\
7 & Fully Connected & 65 & - & - \\
8 & Fully Connected & 3 & - & - \\
\hline
\end{tabular}

The proposed CNN model used three max-pooling layer; the first one receives feature map with size $64 \times 64$ and then applies filter with size $2 \times 2$ with stride 2 . The output of this layer will be $32 \times 32$ feature map. The second max-pooling layer is received feature map with size $32 \times 32$ and then applies filter with size $2 \times 2$ and stride 2 similar to first max-pooling layer. Finally, the third max-pooling layer receives feature map from the last convolutional layer with size $16 \times 16$ and then reduce dimension of received feature map to half. The size of feature map of last max-pooling layer is $8 \times 8$ before input to fully-connected layers.

The CNN model is used two fully-connected layer. The first one is consist of 65 neurons nodes and it is trainable layer. This layer is connected to third max-pooling layer and followed by ReLu activation function layer. The second fully-connected layer is the last layer in CNN model which is responsible of predicted and classify finally output. This layer is consisting of 3 neurons nodes. Also this layer is followed 
by soft-max activation function instead of ReLu activation function. The soft-max activation function is responsible of determining probabilities of output and class that belongs to. In addition, this layer followed by dropout layer which is used to prevent the CNN model from over-fitting. The classification layer of network is containing loss function which compute cross-entropy loss between network predictions and desired output of network. CNN model is used cross-entropy loss function by default and then measures the performance of CNN model during training process. Moreover, cross-entropy loss function is commonly used for classification, specially multi-label classification.

\subsection{Visualization model}

After the CAD3 system detect WBCs by first model and then classify them into its correct label by second model, it need to visualize the WBCs with its correct label on the input image. Furthermore, this model connect the detection model with classification model as shown in Figure 7. Also, the model is not contain learnable parameters and it is not need to training process. The main function of the model is visualizing of WBCs label in input image and give complete statistic of the types of WBCs. Furthermore, the entire input image that contain WBCs with full size $256 \times 256 \times 3$ enter into first model which detect WBCs. The detected WBC images have variant sizes as we mentioned before and the input image contain at least one or more WBC. The extracted cells by detection model store in matrix as stack and then each cells sent to the $\mathrm{CNN}$ model for classification. In other word, after determine the location of each WBC of the input image, each WBC is cropped with its original size and put into stack with keeping of location dimension of each cells. As the second step, the cropped WBCs resize into 64×64 and sent to classification model one by one. After classify each WBC, the size of each WBC image return into its original size and then put into its location with its label. Finally, visualize complete statistic about number and size of each type of WBC and give chart of complete statistic.

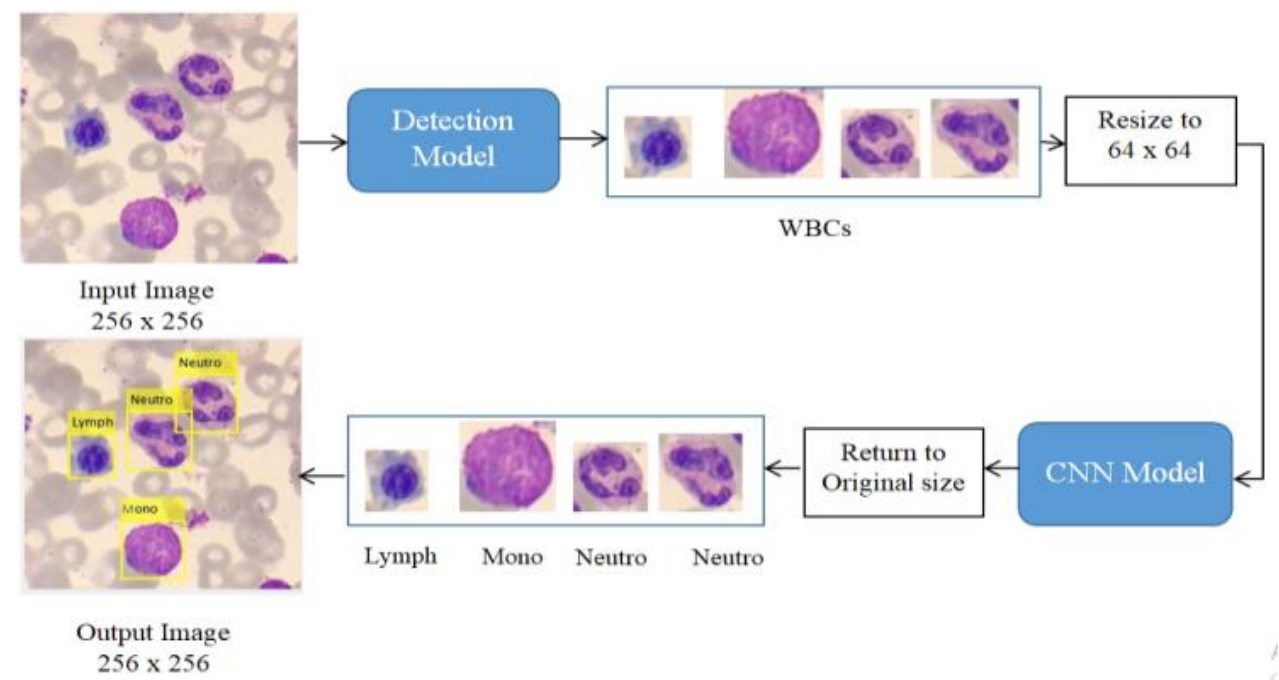

Figure 7. Visualization model process

\section{DATA ACQUISITION AND AUGMENTATION}

The dataset is one of basic ingredients that needed for any deep learning system. The main problem to obtain dataset is difficulty access to data due to data confidentiality, lack of data or other issues. The dataset that used in this study it obtain from private hospital and it prepared to be suitable for CAD3 system. The samples of dataset were acquired from VIN private ospital in Duhok city that situated in Kurdistan region of Iraq. The blood smears of 15 patient of leukemia were took from VIN Hospital lab. The samples took from different ages; adults and children. Moreover, each patient had at least 7 blood films or more. whole films were converted into images. The type of microscope that used is Olympus CX22 light and camera with resolution $12 \mathrm{Mb}$. Moreover, we used lens 100X to visualizing the WBC clearly. The camera fixed on the microscope and then pictures captured for each film as shown in Figure 8. Each film has area that contain more than one pictures. The 360 color images were obtained in high resolution as total of all blood films. The converted images were unsuitable for training the models due to high resolution with dimension 3024x3024 and the images covered with black boarder. Afterward, each of these images are divided into non-overlapping patch to create two dataset one for detection and other for classification. 


\subsection{WBCs detection dataset}

The captured Images divided into non-overlapping patch with size $256 \times 256$. The total images that obtained were 2700 patches and each patch at least contained one or more WBC as shown in Figure 9. In addition, the number of white blood cells for each type was difference after counting each type in dataset.

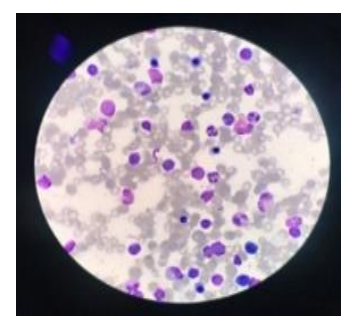

Figure 8. Sample of captured image from microscope with resolution $3024 \times 3024$

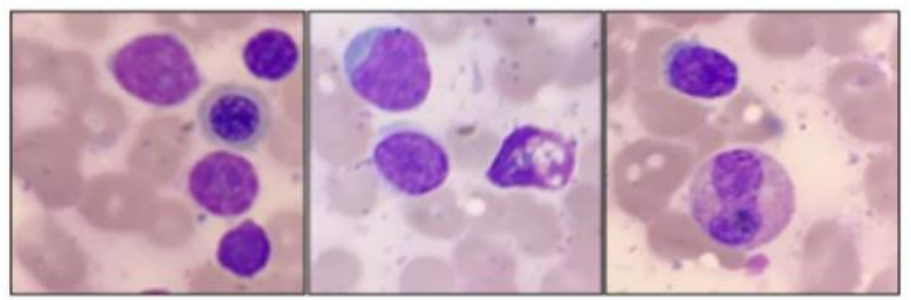

Figure 9. Sample of WBC detection dataset with dimensions $256 \times 256$

Due to inequality among the types of white blood cells, we generated new dataset consist of 1870 color Image with size $256 \times 256$. The data divided into 1500 images which are close in number of WBCs for training and 370 images for testing the model (about $80 \%$ training dataset and 20\% test dataset). Moreover, the new generated dataset called "WBCs detection dataset" which the number of each type of WBCs became close in number as shown in Table 3.

Table 3. Comparison between dataset before and after balancing WBCs types

\begin{tabular}{ccc}
\hline WBC Type & Original Dataset & WBCs Detection Dataset \\
\hline Monocytes & 4117 & 1095 \\
Lymphocyte & 1093 & 1014 \\
Neutrophils & 1092 & 1019 \\
Total Images & 2700 & 1870 \\
\hline
\end{tabular}

\subsection{WBCs classification dataset}

This dataset is generated from the previous dataset "WBCs detection dataset". After creating ground truth bound box and label of all WBC in each image of "WBCs detection dataset". The ground truth it created by "Image Labeler Toolbox" manually. However, the WBCs is cropped depending on its bound box. The new dataset were generated called "WBCs classification dataset" which it contains cropped WBCs. The images in "WBCs classification dataset" had different sizes and then resize into $64 \times 64$ to be suitable for classification model as shown in Figure 10.

Due to imbalanced the WBCs types we chose equal number of each type. In this case the training of classification model will be smoothly. Other WBC images are added into two types to be each type of WBC equal to 1020. Thus, the total images in "WBCs classification dataset" are became 3060 images. The dataset divided into 2448 training and 612 for testing ( $80 \%$ for training and 20\% for testing) as shown in Table 4 .

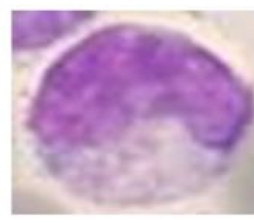

Monocytes

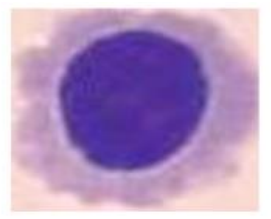

Lymphocyt

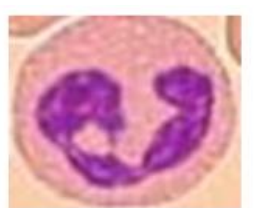

Neutrophils

Figure 10. Samples of WBC classification dataset with dimensions $64 \times 64$

Table 4. Comparison of WBCs classification dataset before and after balancing WBCs types

\begin{tabular}{lcccc}
\hline \multicolumn{1}{c}{ Dataset } & Monocytes & Lymphocyte & Neutrophils & Total Images \\
\hline Before Balance & 1095 & 1014 & 1019 & 3128 \\
Add other image & - & 6 & 1 & 7 \\
After Balance & 1020 & 1020 & 1020 & 3060 \\
\hline
\end{tabular}




\subsection{Data augmentation}

After all cells in WBCs detection ataset defined and created ground truth bound box and label manually. The images in training dataset of WBC detection and classification are few due to lack of data and difficulty obtaining more smears of leukemia patients. However, the data augmentation approach is used to increase the images in dataset. The idea of augmentation data is duplicating images with several variation with preserves the main features key to increase samples for learn model. In this system, WBC detection dataset consist of 1500 images and WBC classification consist of 2448 images. Moreover, randomly rotation (0-90), randomly reflecting in horizontal and vertical axis are used. In addition, color jitter with random values is used which is other data augmented method which used in object.

\section{RESULTS AND DISCUSSION (SYSTEM EVALUATION)}

Two models in CAD3 system need to training process. MATLAB R2020a enviroment with some toolboxes such as deep learning, Image processing and image labeler toolbox ware used to train the proposed CAD3 system on dataset. Moreover, the MATLAB software package installed on DELL laptop with Core i7 single central processing unit (CPU), 8 Giga byte random access memory (RAM) and internal graphic GPU.

However, the detection model trained on "WBCs detection dataset". The 'Adam' optimization algorithm is used to update weights of both models. The detection model trained during 100 epochs with a minimum batch size of 16 and 0.01 initial learning. Each epoch with 93 iterations and the total of iterations were 9300. Each epoch took about 2.5 minutes to get 256 minutes as total time of training. While the second model trained during 50 epochs with a minimum batch size of 64 and 0.01 initial learning. each epoch with 34 iterations and the total of iterations were 1700. In other hand, each epoch took about 1 minutes to be the total time 56.37 minutes. The detection model tested on dataset that consist of 364 images and contain 1557 WBCs. This data did not use in training process and also this dataset has corresponding ground truth. Figure 11 shown sample of test dataset of detection model with corresponding ground truth.

Moreover, an average precision (AP) optimum was achieved by searching over multiple iteration and evaluating the performance of detection model on the appropriate test dataset. The AP and loss value as a function of iteration shown in Figure 12(a) and Figure 12(b) respectively. As we mentioned before, the measured AP value is influenced by both. The optimum AP was obtained at 9300 iterations during 100 epoch with corresponding AP $96 \%$.

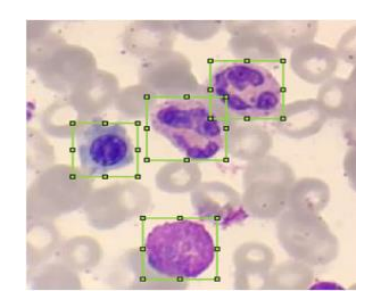

Figure 11. Sample of test dataset

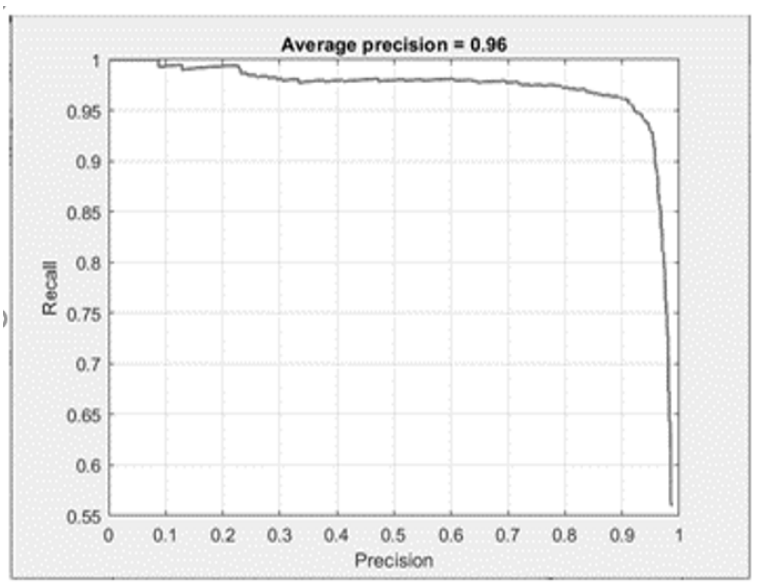

(a)

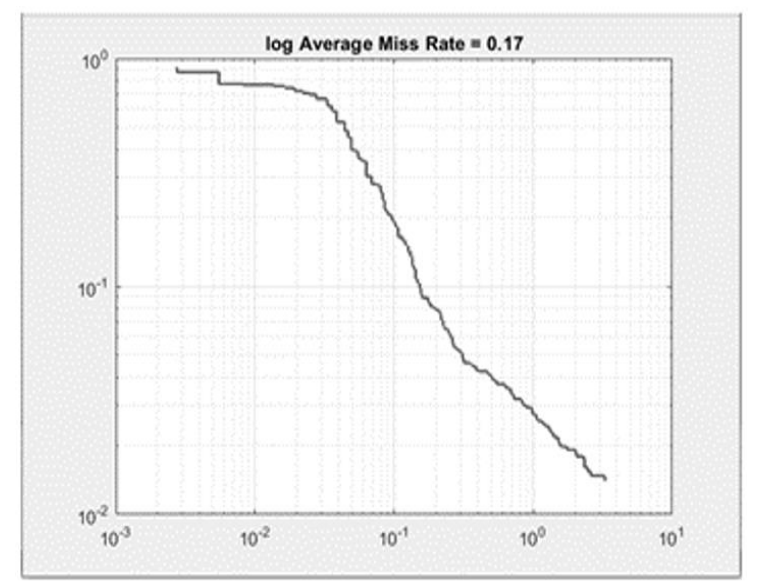

(b)

Figure 12. Detection model of (a) average precision (AP) and (b) average miss rate 
The classification model is tested on the test dataset which consist of $20 \%$ of the "WBCs classification dataset". This dataset contain 612 images and it did not used in training process. However, the Figure 13 illustrates the confusion matrix of classification model on the test dataset. This confusion matrix shows that they are amount of images correctly and incorrectly classified as belonging to target class.

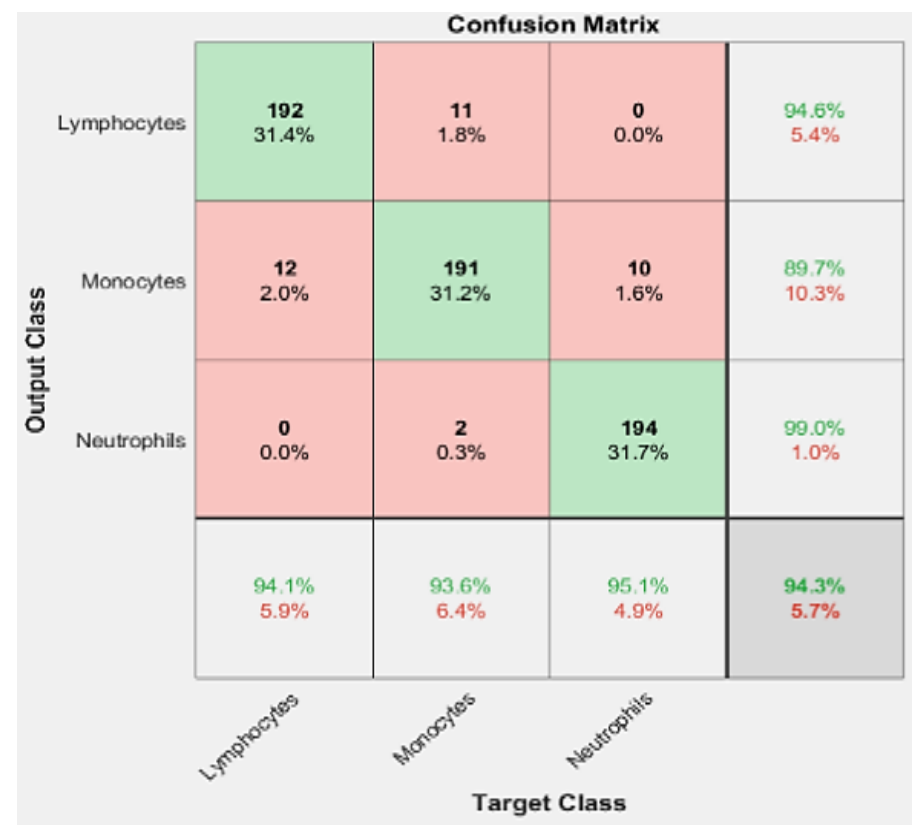

Figure 13. The confusion matrix of classification model

However, the model achieve global accuracy 94.3\%. Table 5 gives the performance evaluation metrics for each class. Where accuracy,

$$
\mathrm{A}=\frac{\mathrm{TP}+\mathrm{TN}}{\mathrm{TP}+\mathrm{FP}+\mathrm{FP}+\mathrm{TN}}
$$

refer to correct classified class with respect to the total number of sample in test dataset. The precision,

$$
\mathrm{P}=\frac{\mathrm{TP}}{\mathrm{TP}+\mathrm{FP}}
$$

is the ratio of images correctly classified for each class divided to the total number of images (correctly or incorrectly) classified to same class. The recall or sensitivity,

$$
\mathrm{R}=\frac{\mathrm{TP}}{\mathrm{TP}+\mathrm{FN}}
$$

is the ratio of images correctly classified for each class with respect to total image that are actually belong to the same class. Finally, the specificity calculate as (4).

$$
\mathrm{S}=\frac{\mathrm{TN}}{\mathrm{FN}+\mathrm{FP}}
$$

Is gives the ratio of images that belong to other class and correctly classified to other class.

The result of visualization model is the final report of the CAD3 system which visualize each class with its label and gives the number of each class as shown in Figure 14(a) as input and Figure 14(b) as output. In addition, the model gives bar chart about information of size for each type cells as illustration in Figure 15.

Table 5. The performance of the classification model on the test dataset

\begin{tabular}{lcccc}
\hline Classification Model & A & P & R & S \\
\hline Lymphocytes & 96.2 & 94.6 & 94.1 & 97.1 \\
Monocytes & 94.3 & 89.7 & 93.6 & 96.7 \\
Neutrophils & 98.0 & 99.0 & 95.1 & 97.6 \\
\hline
\end{tabular}




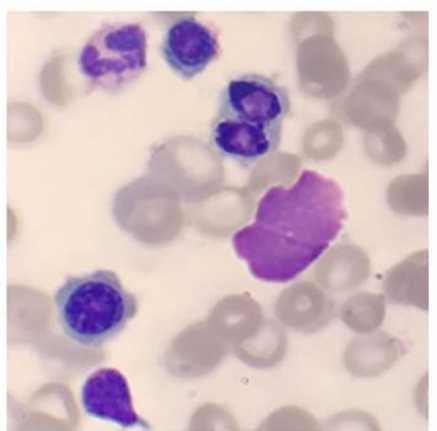

(a)

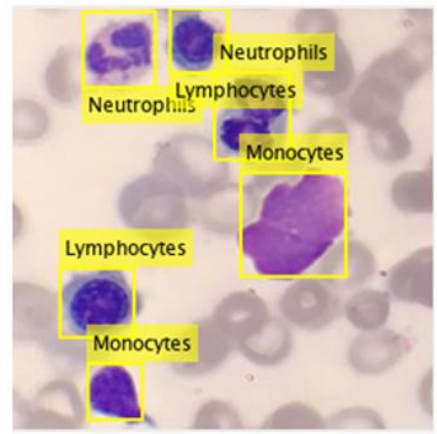

(b)

Figure 14. The localization of WBC with correct label are (a) input image and (b) output image

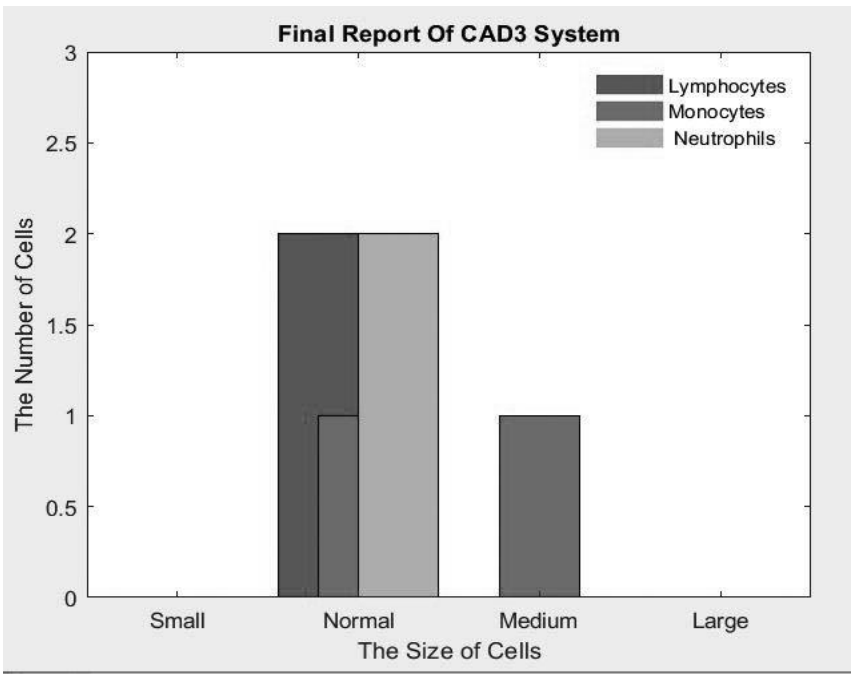

Figure 15. Final report of CAD3

The other objective of CAD3 is proved its ability to perform accurate detection and classification of leukocytes and each model in CAD3 evaluated individually. The detection model achieved high performance on the "WBCs detection dataset" and also the proposed detection model achieved high AP on the other dataset. The Table 6 shows evaluation and comparison of detection model.

Table 6. Evaluation of detection model and comparison with other some systems

\begin{tabular}{ccc}
\hline System & Dataset & Average Precision (AP) \\
\hline YOLO [45] & BCCD & $62 \%$ \\
Proposed Detection Model & BCCD & $98 \%$ \\
Proposed Detection Model & Detection Dataset & $96 \%$ \\
\hline
\end{tabular}

Moreover, Also the classification model evaluated by some important measurements such as global accuracy, precision, recall and specificity. The model achieved high accurate classification of leukocytes. In other side, the classification model achieved high accuracy on the other dataset. The evaluation and comparison of the proposed model with other one shown in Table 7.

Table 7. Evaluation of classification model and comparison with other systems

\begin{tabular}{ccc}
\hline System & Dataset & Accuracy \\
\hline CNN AlexNet [46] & ALL-DB1 & $96.06 \%$. \\
Proposed Classification Model & ALL-DB1 & $97 \%$ \\
Proposed Classification Model & Detection Dataset & $94.3 \%$ \\
\hline
\end{tabular}




\section{CONCLUSION}

The proposed CAD3 system relies on a deep learning by using YOLO v2 and CNN, and it has shown the ability to detect and classify the leukocytes in leukemia. The proposed CAD3 system is capable of achieving perfect results in accuracy and gives perfect report contain important information about the treatment effective on state of leukemia patient. The final report includes the number and size of each type of WBC which not refer by other systems. This study has reached the main tasks of improving the high performance of CAD3 by dividing the main task into sub-task by using simple architectures for each subtask. The proposed CAD3 system trained and tested on data took directly from microscope without any preprocessing or traditional segmentation on the data unlike other system that relies on the preprocessing, segmentation or using filters as basic step before fed the data into system. In other hand, it is important to highlight on other tasks such as creating and preparing dataset which includes two sub-dataset; "detection dataset" and "classification dataset". The preparing of dataset is itself a difficulty and it takes effort. Finally, The CAD3 system has proven its ability to achieve high accuracy on other dataset.

\section{REFERENCES}

[1] M. Togacar, B. Ergen, and M. E. Sertkaya, "Subclass separation of white blood cell images using convolutional neural network models," Elektronika. Ir Elektrotechnika, vol. 25, no. 5, pp. 63-68, Oct. 2019, doi: 10.5755/j01.eie.25.5.24358.

[2] K. AL-Dulaimi, J. Banks, V. Chandran, and I. Tomeo-Reyes, "Classification of white blood cell types from microscope images: techniques and challenges," in book: Microscopy Science: Last Approaches on Educational Programs and Applied Research, Formatex Research Center, Spain p. 10, Nov. 2018.

[3] A. Khvostikov, K. Aderghal, J. Benois-Pineau, A. Krylov, and G. Catheline, "3D CNN-based classification using sMRI and MDDTI images for Alzheimer disease studies," ArXiv Prepr. 2018 ArXiv:1801.05968.

[4] D. Kumar et al., "Automatic detection of white blood cancer from bone marrow microscopic images using convolutional neural networks," IEEE Access, vol. 8, pp. 142521-142531, 2020, doi: 10.1109/ACCESS.2020.3012292.

[5] Z. Shao, W. Wu, Z. Wang, W. Du, and C. Li, "SeaShips: A large-scale precisely annotated dataset for ship detection," IEEE Transactions on Multimedia, vol. 20, no. 10, pp. 2593-2604, 2018, doi: 10.1109/TMM.2018.2865686.

[6] R. Girshick, J. Donahue, T. Darrell, and J. Malik, "Rich feature hierarchies for accurate object detection and semantic segmentation," ArXiv13112524 Cs, Oct. 2014, [Online]. Available: http://arxiv.org/abs/1311.2524.

[7] C. C. Aggarwal, "Neural Networks and Deep Learning," A Textbook, Cham: Springer International Publishing, 2018. doi: 10.1007/978-3-319-94463-0.

[8] J. Pedoeem and R. Huang, "YOLO-LITE: A real-time object detection algorithm optimized for Non-GPU Computers," ArXiv181105588 Cs, Nov. 2018, [Online]. Available: http://arxiv.org/abs/1811.05588.

[9] C. Mishra and D. L. Gupta, "Deep machine learning and neural networks: an overview," IAES International Journal of Artificial Intelligence IJ-AI, vol. 6, no. 2, p. 66, Jun. 2017, doi: 10.11591/ijai.v6.i2.pp66-73.

[10] M. Delgado-Ortet, A. Molina, S. Alférez, J. Rodellar, and A. Merino, "A deep learning approach for segmentation of red blood cell images and malaria detection," Entropy, vol. 22, no. 6, p. 657, Jun. 2020, doi: 10.3390/e22060657.

[11] N. Bibi, M. Sikandar, I. Ud Din, A. Almogren, and S. Ali, "IoMT-Based automated detection and classification of leukemia using deep learning," Journal Healthcare Engineering, vol. 2020, pp. 1-12, Dec. 2020, doi: 10.1155/2020/6648574

[12] I. Mahmood and A. M. Abdulazeez, "The role of machine learning algorithms for diagnosing diseases," Journal of Applied Science and Technology Trends, vol. 2, Mar. 2021, doi: 10.38094/jastt20179.

[13] L. Jiao et al., "A survey of deep learning-based object detection," IEEE Access, vol. 7, pp. 128837-128868, 2019, doi: 10.1109/ACCESS.2019.2939201.

[14] S.-H. Han, K. W. Kim, S. Kim, and Y. C. Youn, "Artificial neural network: understanding the basic concepts without mathematics," Dement. Neurocognitive Disord, vol. 17, no. 3, p. 83, 2018, doi: 10.12779/dnd.2018.17.3.83.

[15] F. Sultana, A. Sufian, and P. Dutta, "Advancements in Image Classification using Convolutional Neural Network," 2018 Fourth International Conference Research in Computational Intelligence and Communication Network ICRCICN, Nov. 2018, pp. 122129, doi: 10.1109/ICRCICN.2018.8718718.

[16] A. Bazaga, M. Roldán, C. Badosa, C. Jiménez-Mallebrera, and J. M. Porta, "A Convolutional Neural Network for the automatic diagnosis of collagen VI-related muscular dystrophies," Applied Soft Computing, vol. 85, p. 105772, Dec.2019, doi: 10.1016/j.asoc.2019.105772.

[17] S. Shafique and S. Tehsin, "Acute lymphoblastic leukemia detection and classification of its subtypes using pretrained deep convolutional neural networks," Technology in Cancer Research Treatment, vol. 17, pp. 1-7, Jan. 2018, doi: $10.1177 / 1533033818802789$.

[18] S. Shamy and J. Dheeba, "A Research on Detection and Classification of Breast Cancer using k- means GMM \& CNN Algorithms," International Journal Engineering and Advanced Technology, vol. 8, no. 6S, pp. 501-505, Sep. 2019, doi: 10.35940/ijeat.F1102.0886S19.

[19] M. Yildirim and A. Çinar, "Classification of White Blood Cells by Deep Learning Methods for Diagnosing Disease," Revue d'Intelligence Artificielle, vol. 33, no. 5, pp. 335-340, Nov. 2019, doi: 10.18280/ria.330502.

[20] M. Jae Lim, D. E. Kim, D. K. Chung, H. Lim, and Y. M. Kwon, "Deep Convolution Neural Networks for Medical Image Analysis," International Journal of Engineering \& Technology., vol. 7, no. 3.33, p. 115, Aug. 2018, doi: 10.14419/ijet.v7i3.33.18588.

[21] G. Liang, H. Hong, W. Xie, and L. Zheng, "Combining Convolutional Neural Network With Recursive Neural Network for Blood Cell Image Classification,” IEEE Access, vol. 6, pp. 36188-36197, 2018, doi: 10.1109/ACCESS.2018.2846685.

[22] I. Chakroun, T. Haber, and T. J. Ashby, "SW-SGD: The Sliding Window Stochastic Gradient Descent Algorithm," Procedia Computer Science, vol. 108, pp. 2318-2322, 2017, doi: 10.1016/j.procs.2017.05.082.

[23] H. S. Yahia and A. M. Abdulazeez, "Medical Text Classification Based on Convolutional Neural Network: A Review," International Journal Science Business, vol. 5, no. 3, pp. 27-41, 2021.

[24] D. Zeebaree, A. M. Abdulazeez, D. A. Zebari, H. Haron, and H. Nuzly, "Multi-level fusion in ultrasound for cancer detection based on Uniform LBP Features," Computers Materials \& Continua, vol. 66, pp. 3364-3382, Dec. 2020, doi: $10.32604 / \mathrm{cmc} .2021 .013314$. 
[25] Y. Xiao, X. Wang, P. Zhang, F. Meng, and F. Shao, "Object Detection Based on Faster R-CNN Algorithm with Skip Pooling and Fusion of Contextual Information," Sensors, vol. 20, no. 19, p. 5490, Sep. 2020, doi: 10.3390/s20195490.

[26] K. Gaurav, A. K. Sahoo, and S. K. Mishra, "Nonlinear System Identification Using Functional Link Multilayer Perceptron Artificial Neural Networks," International Journal of Engineering Research, vol. 10, no. 44, p. 6, 2015.

[27] D. A. Zebari, D. Q. Zeebaree, A. M. Abdulazeez, H. Haron, and H. N. A. Hamed, "Improved Threshold Based and Trainable Fully Automated Segmentation for Breast Cancer Boundary and Pectoral Muscle in Mammogram Images," IEEE Access, vol. 8, p. 1, Nov. 2020, doi: 10.1109/ACCESS.2020.3036072.

[28] Z.-Q. Zhao, P. Zheng, S. Xu, and X. Wu, "Object Detection with Deep Learning: A Review," ArXiv180705511 Cs, Apr. 2019, Accessed: Mar. 10, 2021. [Online]. Available: http://arxiv.org/abs/1807.05511.

[29] R. L. Galvez, A. A. Bandala, E. P. Dadios, R. R. P. Vicerra, and J. M. Z. Maningo, "Object Detection Using Convolutional Neural Networks," in TENCON 2018 - 2018 IEEE Region 10 Conference, Jeju, Korea (South), Oct. 2018, pp. 2023-2027. doi: 10.1109/TENCON.2018.8650517.

[30] F. Yousefian, T. Banirostam, and A. Azarkeivan, "Prediction Thalassemia Based on Artificial Intelligence Techniques: A Survey," International Journal of Advance Reseach in. Computer and Communication Engineering, vol. 6, no. 8, p. 7, Aug. 2017. doi: 10.17148/IJARCCE.2017.6847.

[31] P. Alves-Oliveira, P. Arriaga, A. Paiva, and G. Hoffman, "Guide to build YOLO, a creativity-stimulating robot for children," HardwareX, vol. 6, p. e00074, Oct. 2019, doi: 10.1016/j.ohx.2019e00074.

[32] S. Sasikala, M. Bharathi, and B. R. Sowmiya, "Lung Cancer Detection and Classification Using Deep CNN," International Journal of innovative Technology and Exploring Engineering, vol. 8, no. 2, p. 4, 2018.

[33] Mohana and H. V. R. Aradhya, "Object Detection and Classification Algorithms using Deep Learning for Video Surveillance Applications," International Journal of innovative Technology and Exploring Engineering, vol. 8, no. 8, p. 10, 2019.

[34] C. N. The and D. Shashev, "Methods and Algorithms for Detecting Objects in Video Files," MATEC Web of Conferences, 2018, vol. 155, p. 01016, doi: 10.1051/matecconf/201815501016.

[35] J. Redmon and A. Farhadi, "YOLO9000: Better, Faster, Stronger," in 2017 IEEE Conference on Computer Vision and Pattern Recognition (CVPR), Jul. 2017, pp. 6517-6525. doi: 10.1109/CVPR.2017.690.

[36] S. Khan, H. Rahmani, S. A. A. Shah, and M. Bennamoun, "A Guide to Convolutional Neural Networks for Computer Vision," Synthesis Lectures on Computer Vision., vol. 8, no. 1, pp. 1-207, Feb. 2018, doi: 10.2200/S00822ED1V01Y201712COV015.

[37] I. Rahadi, M. Choodoung, and A. Choodoung, "Red blood cells and white blood cells detection by image processing," Journal of Physics: Conference Series, May 2020, vol. 1539, p. 012025, doi: 10.1088/1742-6596/1539/1/012025.

[38] N. Ghane, A. Vard, A. Talebi, and P. Nematollahy, "Segmentation of White Blood Cells From Microscopic Images Using a Novel Combination of K-Means Clustering and Modified Watershed Algorithm," Journal of Medical Signals \& Sensors., vol. 7, no. 2, p. 92, 2017, doi: 10.4103/2228-7477.205503.

[39] M. Rieder and R. Verbeet, "Robot-human-learning for robotic picking processes," Hamburg International Conference of Logistics., Sep. 2019, doi: 10.15480/882.2466.

[40] C. Zhang, X. Xu, and D. Tu, "Face Detection Using Improved Faster RCNN," Feb. 2018.

[41] S. N. M. Safuan, M. R. Md Tomari, and W. N. Wan Zakaria, "White blood cell (WBC) counting analysis in blood smear images using various color segmentation methods," Measurement, vol. 116, pp. 543-555, Feb. 2018, doi: 10.1016/j.measurement.2017.11.002.

[42] H. A. Aliyu, R. Sudirman, M. A. Abdul Razak, and M. A. Abd Wahab, "Red Blood Cell Classification: Deep Learning Architecture Versus Support Vector Machine," in 2018 2nd International Conference on BioSignal Analysis, Processing and Systems (ICBAPS), Kuching, Jul. 2018, pp. 142-147. doi: 10.1109/ICBAPS.2018.8527398.

[43] T. Pansombut, S. Wikaisuksakul, K. Khongkraphan, and A. Phon-on, "Convolutional Neural Networks for Recognition of Lymphoblast Cell Images," Computational Intelligence and Neuroscience., vol. 2019, pp. 1-12, Jun. 2019, doi: $10.1155 / 2019 / 7519603$.

[44] J. Hung et al., "Applying Faster R-CNN for Object Detection on Malaria Images," HHS Public Access p. 15, Apr. 2018.

[45] M. M. Alam and M. T. Islam, "Machine learning approach of automatic identification and counting of blood cells," Healthcare Technology Letters, vol. 6, no. 4, pp. 103-108, Aug. 2019, doi: 10.1049/htl.2018.5098.

[46] T. T. P. Thanh, C. Vununu, S. Atoev, S.-H. Lee, and K.-R. Kwon, "Leukemia Blood Cell Image Classification Using Convolutional Neural Network," International Journal of Computer Theory and Engineering, vol. 10, no. 2, pp. 54-58, 2018, doi: 10.7763/IJCTE.2018.V10.1198.

\section{BIOGRAPHIES OF AUTHORS}

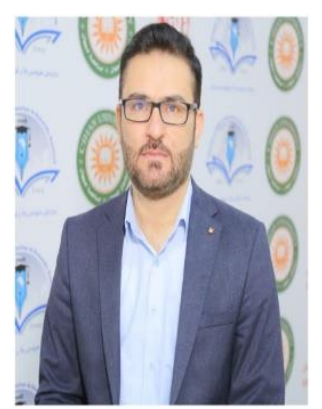

Shakir Mahmood Abas (iD) SC SC Currently, he is a manager of presidency office of Cihan University-Duhok and he work as lecturere at Computer Sciences department at Cihan University-Duhok, Kurdistan region, Iraq. He is a MSc degree in Information Technology (IT) from Duhok Polytechnic University, Iraq. in 2021, and B.S. degree in Computer Science from the University of Duhok, in 2010. He is a author of 5 articles of which most of them indexed in Scopus. Research interests include artificial neural networks, machine learning, deep learning, medical image analysis, and image processing. He can be contacted at email: Shakr.abbas@duhokcihan.edu.krd. 

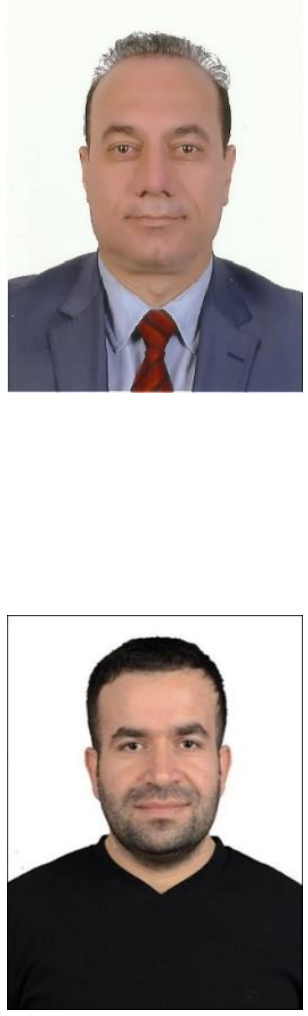

Adnan Mohsin Abdulazeez (iD IS SC P received the B.Sc. degree in electrical and electronic engineering, the M.Sc. degree in computer and control engineering, and the $\mathrm{Ph} . \mathrm{D}$. degree in computer engineering. He is currently the President of Duhok Polytechnic University and a Professor of computer engineering and science. He held the position of the Dean of the Duhok Technical Institute for several years. Before that, he was assigned as the Head of several scientific departments and committees in various public and private universities in Kurdistan Region and Iraq. He has been awarded the title of a professor since 2013. He is keen to carry out his administrative and academic responsibilities simultaneously, and therefore he supervised and still supervises a large number of master and doctoral students. In addition, he focuses his attention on publishing scientific researches in valuable international scientific journals, so he has published many researches in these journals. His research interests include intelligence systems, soft computing, multimedia, network security and coding, and FPGA implementation. He has created and led various research groups, as well as urged and encouraged them to publish research in different international journals. He is a Reviewer for several accredited international journals. He can be contacted at email: Adnan.Mohsin@dpu.edu.krd.

Diyar Qader Zeebaree (D) SC SC P He currently work at the Duhok Polytechnic University, Iraq. He received $\mathrm{PhD}$ degree in Computer Science from University Technology Malaysia (UTM), in 2020, MSc degree in Computer Information Systems (CIS) from Near East University, North of Cyprus, Turkey, in 2014, and B.S. degree in Computer Science from the University of Nawroz, in 2012. He is a author of one book and more than 52 articles of which most of them indexed in Web of Science (WOS) and Scopus. He work as a reviewer of IEEE access and CMC journal and others. Research interests include artificial neural networks, machine learning, deep learning, medical image analysis, and image processing. He has a recipient of Best Symposium Paper Award in the IEEE-International Conference on Advanced Science and Engineering (ICOASE), in 2019. He can be contacted at email: Dqszeebaree@dpu.edu.krd. 